\title{
Validity and reliability of Resource Utilization Groups (RUG-III) in Finnish long-term care facilities
}

\author{
Magnus A. Björkgren ${ }^{1}$, Unto Häkkinen ${ }^{2}$, U. Harriet Finne-Soveri ${ }^{3}$ and Brant E. Fries ${ }^{4}$ \\ ${ }^{1}$ National Research and Development Centre for Welfare and Health, Health Services Research Unit, Helsinki and \\ Chydenius Institute, University of Jyväskylä, Kokkola, Finland, ${ }^{2}$ National Research and Development Centre for Welfare \\ and Health, Health Services Research Unit, Helsinki, ${ }^{3}$ Koskela Hospital, Helsinki, Finland, ${ }^{4}$ Institute of Gerontology and \\ School of Public Health, The University of Michigan and Ann Arbor VA Medical Center, Ann Arbor, Michigan, USA
}

Scand J Public Health 1999; 27: 228-234

\begin{abstract}
Resource Utilization Groups, Version III (RUG-III) is a case-mix system developed in the USA for classification of long-term care residents. This paper examines the validity and reliability of an adapted 22-group version of RUG-III (RUG-III/22) for use in long-term care facilities in Finland. Finnish cost weights for RUG-III/22 groups are calculated and different methods for their computation are evaluated. The study sample (1,964 residents) was collected in 1995 - 96 from ten long-term care facilities in Finland. RUG-III/22 alone explained 38.2\% of the variance of total patient-specific (nursing + auxiliary staff) per diem cost. Resource use within RUG groups was relatively homogeneous. Other predictors of resource use included age, gender and length of stay. RUG-III/ 22 also met the standard for good reliability (i.e. a kappa value of 0.6 or higher) for crucial classification items, such as activities of daily living and high correlation between assessments based on relative cost.
\end{abstract}

Key words: case-mix, long-term care, nursing homes, patient classification, payment systems, resource use.

Magnus Björkgren, Chydenius Institute, Pitkänsillankatu 1-3, FIN-67100 Kokkola, Finland. Tel: Tel: +358 6 8294226, fax: +35868294202.E-mail: magnus.bjorkgren@chydenius.fi

\section{INTRODUCTION}

Case-mix classification systems describe resource utilization in healthcare. In acute care hospitals, case-mix measurement is based largely on applications of Diagnosis-Related Groups (DRGs) (1). DRGs have been implemented in the Nordic countries for management and policymaking throughout the 1990s, most recently as a payment method in health districts in southern Finland. In early applications of DRGs in the USA, it became clear that a system predicting cost of care episodes would not apply to long-term care provided to the elderly. In long-term care the variability of length of stay, and thereby of episode cost, is too great to be practical for case-mix classification and payment. Thus, another system, Resource Utilization Groups (RUGs), was developed for measuring case-mix of nursing home residents $(2-4)$. It is based on a measure of per diem resource use.

In the USA, RUG Version III (RUG-III) forms the basis for prospective payment of publicly reimbursed nursing home care. In USA Medicaid programs (public health insurance for persons with low income) accounting for about half of all nursing home expenditures, RUG-III is used for payment in one third of US states.
In the federal Medicare program (public health insurance for persons over 65 years), covering nearly $10 \%$ of total nursing home costs, RUG-III is being adopted for national prospective payment. RUG classifications have been found to be valid across nations and healthcare systems. RUG-III has been tested successfully in long-term care facilities in England and Wales, Japan and Spain (5-7). An earlier version, RUG Version II, has been validated in Sweden and The Netherlands (8, 9). RUG-III is based on information in the Resident Assessment Instrument (RAI) and its Minimum Data Set (MDS). The RAI is a standardized assessment instrument implemented nationwide in the USA since 1990 for improving care planning and quality of care $(10,11)$. The RAI and the RUG-III classification are systems currently being tested in all the Nordic countries. In Iceland the RAI has been mandated by the government for use in all nursing homes since 1996 (12).

We assess here the validity and reliability of an adapted, 22-group version of RUG-III (RUG-III/22) in long-term care facilities in Finland. By validity, we mean the criterion-related or predictive validity of the classification system to explain resource use in longterm care. Reliability is measured by the inter-rater 
agreement of classifying a resident into a specific RUG-III/22 group. The aim is also to derive Finnish cost weights, case-mix index, for RUG-III/22 to create a basis for a national case-mix measurement, and resource allocation in long-term care, e.g. for determining staffing levels in facilities and for developing payment methods and as an output measure in studies of productivity in long-term care facilities.

In Finland, local municipalities have the primary responsibility for providing institutional long-term care for the elderly. There are two basic settings for institutional care: residential homes and health centre hospitals (13). Residential homes are the most common form of long-term care facility, traditionally owned, managed, and administered through social services of single municipalities. Health centre hospitals provide care for the sickest and frailest long-term residents, although they also provide some acute care. They are owned and managed either by single municipalities or through federations of municipalities.

\section{RESOURCE UTILIZATION GROUPS, VERSION III (RUG-III)}

RUG classifications, first developed in the USA in the mid-1980s, were produced to meet statistical, clinical and incentives criteria $(2-4)$. The major statistical criteria included the power of the classification system to explain the cost of caring for long-term care residents, and the homogeneity of cost within the classification groups. The RUG system was also constructed to make clinical sense: that residents within groups had clinical affinity. Patient characteristics used to classify residents, were chosen that could be reliably assessed and which would provide incentives for appropriate care. Where possible, measures of need for service were used rather than the provision of the service itself.

The latest version, RUG-III, was derived based on a sample of 7,658 residents in 203 nursing homes in seven US states (4). The system has been recently validated and slightly revised for the latest version of the MDS (Version 2.0) on an additional sample of over 2,000 residents. RUG-III classifies a resident into one of 44 distinct groups. The system incorporates three dimensions in describing and grouping a resident. The first dimension is represented by seven major clinical categories. These categories are devised as a hierarchy with decreasing cost intensity: 1) special rehabilitation: residents receiving different degrees of physical, occupational, or speech therapy; 2) extensive services: residents with respirator/ventilator care, parenteral feeding suctioning, or tracheostomy; 3) special care: e.g. residents with burns, coma, multiple sclerosis, pressure ulcers stage 3 or 4 , quadriplegia, septicaemia, IV medications, or tube feeding; 4) clinically complex: e.g. residents with aphasia, cerebral palsy, dehydration, hemiplegia, pneumonia, static ulcer, terminal illness, urinary tract infection, dialysis, or four or more physician visits per month; 5) impaired cognition: e.g. residents with impaired decision-making, orientation problems, short-term memory problems; 6) behaviour problems: e.g. residents with physical abuse, verbal abuse, or wandering; and 7) reduced physical functions: residents who do not meet the conditions of earlier categories.

The second dimension, used to subdivide the major categories, is based on an ADL (Activities of Daily Living) Index, a summary measure of functional capability in four ADLs: bed mobility, transfers, eating and toilet use. The ADL Index ranges from 4 to 18, the lowest value (4) indicating independence in all four ADLs, and the highest value total dependency in these same four ADLs. The third dimension forms tertiary splits in the classification and incorporates particular services; rehabilitation provided by nurses, or problems, presence of depression. Depression is used as tertiary splits in the "clinically complex" category, and "nursing rehabilitation" as tertiary splits in "impaired cognition", "behaviour problems" and "reduced physical functions". Detailed information of the derivation process and RUG-III definitions are provided elsewhere (4).

\section{The RUG-III/22 model}

Early in our empirical analysis it became clear that several groups of the original 44-group RUG-III model would be relatively rare in Finnish patterns of longterm care and the study sample. Thus, we decided to test a reduced model of RUG-III, consisting of only 22 groups. If a reduced model was supported by statistical criteria it would provide more stable estimates of the cost weights. The model we tested reduced the number of groups primarily by collapsing those groups formed by the tertiary splits (depression or nursing rehabilitation). These splits were initially constructed mainly to provide payment incentives, they added only $0.1 \%$ to the variance in cost of care explained by RUGIII. The differences in relative cost between two groups formed by tertiary splits were also minimal (4). Collapsing the tertiary splits reduced the number of groups by a total of 13. Given the low numbers of heavy rehabilitation residents, we decided also to collapse the number of rehabilitation groups from 12 to 3 . The task of providing special rehabilitation to elderly residents is subject to local variation in Finland, and is not only provided by their long-term care facilities. Residents were classified as "special rehabilitation" if they received a weekly total of $45 \mathrm{~min}$ or more, at least four days a week, of physical, occupational or speech 


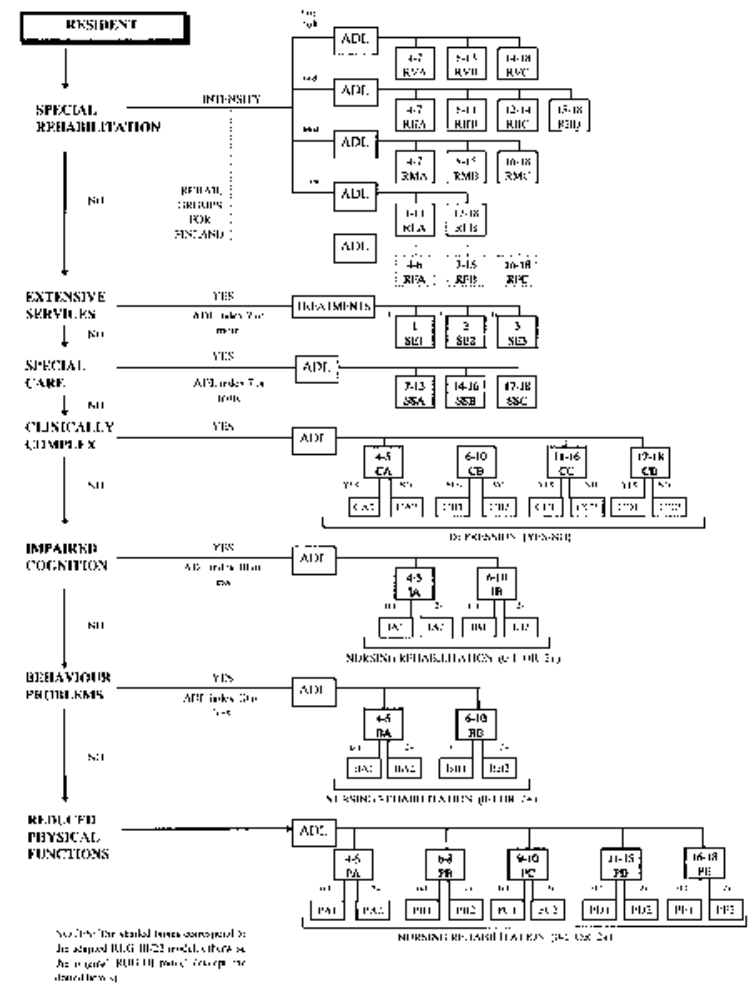

Fig. 1. RUG-III classification (original and adapted RUG-III/22 model).

therapy. The revision of the "special rehabilitation" criteria increased the number of patients classified into this category from 38 to 80 . We split this rehabilitation category into three rehabilitation groups by splitting based on the ADL Index. Beyond this, we made no structural changes to definitions of other RUG-III categories. It should be noted that adopting a reduced model does not mean that residents cannot be classified according to the 44-group criteria if so desired. The advantages of our reduced model is that less information is required to classify a resident, and that the prediction of resource use is only marginally different. The classification algorithm for the original 44-group model and the adapted 22-group model with all of these changes are shown in Figure 1.

\section{MATERIALS AND METHODS}

\section{Reliability analysis}

We tested the Inter-rater reliability of the MDS items necessary to classify a resident into a distinct RUGIII/ 22 group using data from two facilities: one residential home and one health centre hospital. A total of 41 subjects were assessed by two assessors operating independently who knew the resident equally well. Generally the assessors were a personal nurse and another nurse working on the same ward. An addi- tional 32 residents were assessed comparing a personal nurse and an outsider evaluator, nurse from other facility or building. Both samples represented patients with a range of care needs.

Kappa statistics and Pearson correlations were used to represent the congruence between the judgements of two assessors. Kappa coefficients were calculated on RUG-III/22 group items with an average prevalence of between approximately $10 \%$ and $90 \%$. A kappa of 1.0 denotes perfect agreement. According to Fleiss, kappa values lower than 0.40 indicate poor reliability, $0.40-0.75$ adequate or good agreement, and values over 0.75 excellent agreement (14). We used weighted kappa statistics for categorical variables and simple kappa statistics for dichotomous variables; the kappa statistic then corresponds to the Spearman-Brown Intraclass Correlation Coefficient (ICC) (15).

\section{Validity analysis and deriving cost weights}

The validity analysis and calculation of cost weights were based on a sample of 1,964 residents selected from 67 wards in ten long-term care facilities in Finland. About $45 \%$ of the sample comprised residents from residential homes, with the remainder from health centre hospitals. More than half of the residents came from two large facilities in the city of Helsinki. Facilities were chosen on a voluntary basis, with a larger share of health centre hospital residents sought to assure adequate representation of heavy care residents. Wards providing care mainly for short-term residents were not included in the sample. All of the facilities operated in urban areas. Data were collected between May 1995 and February 1996. Members of the research group translated the instrument from English to Finnish. It should be noted that the sample is not necessarily representative of all residents in Finnish long-term care facilities, but for this research we only need it to be representative of the types of residents seen in these facilities.

Staff on the wards of eight of the facilities completed an abbreviated MDS assessment including the items necessary to classify each resident into a RUG-III/22 group; in two facilities the full MDS was used. Staff members on a nursing unit/ward recorded their time caring for each resident, both in direct "hands-on" care and other care for the resident, such as charting, care planning meetings, etc. Care times were collected over a 24-h period. Therapist, physician and other auxiliary staff time was recorded over a longer, 7-day, period as such time can be variable over a week, such as a Monday and Thursday rehabilitation schedule. Informal care time provided at the facility by family and friends was included only to the extent it replaced formal care time. To develop a cost measure, care time was weighted by cost using wage rates of respective staff 
Table 1. Reliability estimates of the RUG-III/22 model

\begin{tabular}{lllllll}
\hline & $\begin{array}{l}\text { Number of } \\
\text { residents in } \\
\text { test group }\end{array}$ & $\begin{array}{l}\text { \% agreement } \\
\text { RUG-III/22 } \\
\text { group }\end{array}$ & $\begin{array}{l}\text { \% agreement } \\
\text { RUG-III/22 } \\
\text { clinical } \\
\text { category }\end{array}$ & $\begin{array}{l}\text { Mean kappa } \\
\text { RUG-III/22 } \\
\text { all items }\end{array}$ & $\begin{array}{l}\text { Mean kappa } \\
\text { RUG-III/22 } \\
\text { ADL items }\end{array}$ & $\begin{array}{l}\text { Pearson } \\
\text { correlation } \\
\text { cost weights }\end{array}$ \\
\hline $\begin{array}{l}\text { Personal nurse vs. } \\
\text { personal nurse }\end{array}$ & 41 & $68 \%$ & $78 \%$ & $0.71^{1}$ & 0.73 & 0.93 \\
$\begin{array}{l}\text { Personal nurse vs. } \\
\text { outsider evaluator }\end{array}$ & 32 & $44 \%$ & $69 \%$ & $0.59^{2}$ & 0.68 & 0.78 \\
\hline
\end{tabular}

NOTES: Kappa coefficient $<0.40$ poor agreement, $0.40-0.75$ fair to good agreement, $>0.75$ excellent agreement (Fleiss, 1981).

${ }^{1}$ Based on $44 \%$ of RUG-III $/ 22$ items (prevalence $>10 \%$ ).

${ }^{2}$ Based on $39 \%$ of RUG-III $/ 22$ items (prevalence $>10 \%$ ).

category. Informal care time was weighted using the wage-weight for nursing assistant/aide.

Our tests of the validity of the adapted RUG-III/22 model focused mainly on the statistical criteria, since the clinical and "administrative" criteria were regarded to be the same in the USA and Finland. For fitting RUG-III/22 models we used Analysis of Variance (ANOVA), applying regression analysis. Wageweighted patient specific time served as the dependent variable. Initially, we examined four dependent variables: a wage-weighted time of nursing staff only, and three others adding different categories of auxiliary staff. The results using different compositions of staff care time did not differ greatly. We therefore report here only the results of wage-weighted total patient specific nursing + auxiliary care time. The explanatory power of the system was measured by the percentage of variance in resource use explained by the classification (R-square). Indicator (dummy) variables represented membership in each of the RUG-III/22 groups, the basic independent variables of the analysis. In addition, we evaluated how age, gender, length of stay and ward identifiers increased the fit of the 22-group model. The homogeneity of resource use within groups was measured by each groups' coefficient of variation and by testing the difference between group means. The cost weights for each RUG-III/22 group were calculated by dividing the group mean by total sample mean of wage-weighted patient specific time. Refined cost weights were calculated adjusting for factors found statistically significant in covariate analysis. The adjusted cost weights were calculated based on parameter estimates of RUG-III/22 dummy variables from regression models where the covariates were included.

\section{RESULTS}

\section{Reliability analysis}

Overall, the agreement of two evaluators who knew the residents equally well was slightly better than the agreement of a personal nurse and an outsider evaluator (Table 1). The percentage agreement reported indicates the proportion of residents classified by two evaluators into an identical RUG-III/22 group and main clinical category. For the comparison of two personal nurses, kappa values for each RUG-III/22 item with over $10 \%$ prevalence were statistically significant and greater than 0.40 . The mean kappa 0.71 indicated good agreement. In the comparison of the personal nurse and outsider evaluator, two RUG-III/22 items in memory/recall ability were lower than 0.40 and the kappas of two additional items were not statistically significant. Overall, the mean kappa (0.59) indicated adequate or good agreement. Kappa values for ADL variables indicated high agreement in both test groups (0.73 and 0.68), ADLs are key items in determining the cost intensity of RUG-III/22 groups.

It should be noted that disagreement on a single item often results in classification into a different RUG-III/ 22 group, although not necessarily with a very different resource use. For costing purposes, agreement based on cost weights is of more relevance than agreement on clinical group. The correlation coefficient, in comparison of cost weights from assessments performed by the evaluators who knew the residents equally well, indicated high agreement $(r=0.93)$. The mean cost weights of each pair of assessments was 0.996 and 0.984 , and the difference was not statistically significant. For the other test group, the correlation was somewhat lower $(r=0.78)$, but still acceptable. The outsider evaluator tended to grade a lower disability level than the personal nurse. The mean cost weight for the assessments by personal nurses was 0.945 , while the corresponding mean by outsider evaluators was 0.864 , but again the difference was not statistically significant.

\section{Validity analysis and cost weights}

In the sample used to test the validity of the RUG-III/ 22 model, the median age of the residents was 83 years, 
Table 2. Frequency, coefficient of variation ( $C V$ ), and cost weights by $R U G-I I I / 22$ groups

\begin{tabular}{|c|c|c|c|c|c|c|c|c|}
\hline \multirow[b]{2}{*}{$\begin{array}{l}\text { RUG-III/22 } \\
\text { Group Name }\end{array}$} & \multirow[b]{2}{*}{$\begin{array}{l}\text { Group } \\
\text { code }\end{array}$} & \multirow[b]{2}{*}{$\begin{array}{l}\mathrm{ADL} \\
\text { range }\end{array}$} & \multirow[b]{2}{*}{$\mathrm{N}$} & \multirow{2}{*}{$\begin{array}{l}\mathrm{CV} \\
\text { No } \\
\text { adjustments }\end{array}$} & \multicolumn{4}{|l|}{ Cost weights } \\
\hline & & & & & $\begin{array}{l}\text { No } \\
\text { adjustments }\end{array}$ & $\begin{array}{l}\text { Adjusted } \\
\text { for LOS, } \\
\text { gender, age }\end{array}$ & $\begin{array}{l}\text { Adjusted } \\
\text { for ward }\end{array}$ & $\begin{array}{l}\text { Adjusted for } \\
\text { ward, LOS, } \\
\text { gender, age }\end{array}$ \\
\hline \multicolumn{3}{|l|}{ Special rehabilitation } & 80 & 0.63 & 1.59 & 1.61 & 1.58 & 1.61 \\
\hline REHAB $16-18$ & RFC & $16-18$ & 15 & 0.54 & 2.52 & 2.66 & 2.57 & 2.68 \\
\hline REHAB $7-15$ & RFB & $7-15$ & 41 & 0.50 & 1.62 & 1.67 & 1.64 & 1.69 \\
\hline REHAB 4-6 & RFA & $4-6$ & 24 & 0.47 & 0.98 & 0.86 & 0.86 & 0.78 \\
\hline \multicolumn{3}{|l|}{ Extensive services } & 38 & 0.42 & 1.55 & 1.63 & 1.53 & 1.61 \\
\hline EXTENSIVE 2 & SE2 & & 4 & 0.41 & 1.40 & 1.46 & 1.39 & 1.43 \\
\hline EXTENSIVE 1 & SE1 & & 34 & 0.43 & 1.57 & 1.65 & 1.55 & 1.63 \\
\hline \multicolumn{3}{|l|}{ Special care } & 71 & 0.48 & 1.59 & 1.67 & 1.70 & 1.77 \\
\hline SPECIAL $17-18$ & $\mathrm{SSC}$ & $17-18$ & 37 & 0.44 & 1.87 & 2.00 & 2.00 & 2.11 \\
\hline SPECIAL $14-16$ & SSB & $14-16$ & 22 & 0.42 & 1.31 & 1.36 & 1.42 & 1.46 \\
\hline SPECIAL $7-13$ & SSA & $7-13$ & 12 & 0.42 & 1.26 & 1.25 & 1.30 & 1.28 \\
\hline \multicolumn{3}{|l|}{ Clinically complex } & 829 & 0.52 & 1.10 & 1.11 & 1.10 & 1.11 \\
\hline COMPLEX $17-18$ & $\mathrm{CD}$ & $17-18$ & 252 & 0.38 & 1.31 & 1.35 & 1.35 & 1.39 \\
\hline COMPLEX $11-16$ & $\mathrm{CC}$ & $11-16$ & 283 & 0.43 & 1.26 & 1.30 & 1.25 & 1.28 \\
\hline COMPLEX $6-10$ & $\mathrm{CB}$ & $6-10$ & 129 & 0.66 & 0.90 & 0.88 & 0.90 & 0.88 \\
\hline COMPLEX 4-5 & $\mathrm{CA}$ & $4-5$ & 165 & 0.65 & 0.68 & 0.60 & 0.62 & 0.56 \\
\hline \multicolumn{3}{|l|}{ Impaired cognition } & 76 & 0.71 & 0.57 & 0.54 & 0.56 & 0.53 \\
\hline IMPAIRED $6-10$ & IB & $6-10$ & 30 & 0.55 & 0.76 & 0.74 & 0.70 & 0.67 \\
\hline IMPAIRED $4-5$ & IA & $4-5$ & 46 & 0.79 & 0.45 & 0.40 & 0.47 & 0.43 \\
\hline \multicolumn{3}{|l|}{ Behaviour problems } & 191 & 0.78 & 0.65 & 0.62 & 0.67 & 0.64 \\
\hline BEHAVIOUR $6-10$ & $\mathrm{BB}$ & $6-10$ & 78 & 0.62 & 0.90 & 0.90 & 0.92 & 0.92 \\
\hline BEHAVIOUR 4-5 & $\mathrm{BA}$ & $4-5$ & 113 & 0.82 & 0.48 & 0.42 & 0.50 & 0.45 \\
\hline \multicolumn{3}{|c|}{ Reduced physical functions } & 679 & 0.67 & 0.86 & 0.85 & 0.85 & 0.83 \\
\hline PHYSICAL 16-18 & PE & $16-18$ & 168 & 0.34 & 1.29 & 1.34 & 1.27 & 1.31 \\
\hline PHYSICAL $11-15$ & PD & $11-15$ & 152 & 0.47 & 1.15 & 1.19 & 1.15 & 1.18 \\
\hline PHYSICAL $9-10$ & $\mathrm{PC}$ & $9-10$ & 21 & 0.46 & 1.04 & 1.02 & 0.99 & 0.97 \\
\hline PHYSICAL 6-8 & $\mathrm{PB}$ & $6-8$ & 75 & 0.56 & 0.77 & 0.76 & 0.71 & 0.69 \\
\hline PHYSICAL 4-5 & $\mathrm{PA}$ & $4-5$ & 263 & 0.84 & 0.42 & 0.34 & 0.42 & 0.36 \\
\hline All* & & & 1964 & 0.65 & 1.00 & 1.00 & 1.00 & 1.00 \\
\hline
\end{tabular}

*The adjusted cost weights are calibrated the same manner as the non-adjusted, i.e. the mean cost weight of all residents was set to 1.00 .

females accounted for $77.5 \%$, and the mean length of stay for the residents was 865 days. The mean staff time caring for residents was $76.4 \mathrm{~min}$ per 24 -h period, 72.3 min by nursing staff, and 4.1 min by auxiliary staff. The mean informal care time, substituting nursing care, was 8.9 min per $24 \mathrm{~h}$. The case-mix adjusted mean staff time was lower than that of other validation studies. (Case-mix adjusted mean staff time per 24-h period using US cost weights, was 86 min for Finland, 92 min for Japan, 126 min for the USA and $148 \mathrm{~min}$ for England and Wales.) The average time for completing the MDS assessment form including only RUG-III items was $14 \mathrm{~min}$.

The distribution of residents into RUG-III/22 main clinical categories and final groups are presented in Table 2. The two largest clinical categories were "clinically complex" (829 residents or $42 \%$ ) and "reduced physical functions" (679 or $35 \%)$. These two categories have similarly been the largest in other validation studies $(5-7)$. Residents were classified into 21 of 22 pos- sible groups. The 22-group model explained $38.2 \%$ of the variance of total wage-weighted patient specific time (Table 3). Our explanatory power was slightly higher than that in the England and Wales study $(35.6 \%)$, which used a similar sample size, but was lower than the variance explained in the Japanese study $(43.8 \%)$, and the original US derivation $(55.5 \%)$. The high variance explanation in the US study has been shown to be partly due to input from rehabilitation staff, the variance explanation of nursing staff cost alone was $41 \%$ (2). Adding dummy variables for wards to the RUG-III/22 model increased the explanatory power to $49.9 \%$. Dummy variables for gender, age and length of stay (LOS) were all found statistically significant when added to the basic model. Adjusting for case-mix, residents with LOS of less than two weeks used about $22 \min (29 \%)$ more care time than others, females used about seven min $(10 \%)$ more than males, and residents under the age of 75 used six min $(8 \%)$ more than those older. Interactions between gender, 
Table 3. Variance explanation and covariate estimates of RUG-III/22 models

\begin{tabular}{|c|c|c|c|c|}
\hline & RUG-III/22 & $\begin{array}{l}\text { RUG-III/22 } \\
+ \text { LOS, } \\
\text { gender, age }\end{array}$ & $\begin{array}{l}\text { RUG-III/22 } \\
+ \text { ward }\end{array}$ & $\begin{array}{l}\text { RUG-III/22 } \\
\text { + ward LOS, } \\
\text { gender, age }\end{array}$ \\
\hline \multicolumn{5}{|l|}{ Covariate estimates } \\
\hline Length of stay (LOS) $<2$ weeks & & $22.1^{* * *}$ & & $13.8^{* * *}$ \\
\hline Female & & $7.9^{* * *}$ & & $7.4^{* * *}$ \\
\hline Age $<75$ years & & $6.0^{*}$ & & $6.1 * *$ \\
\hline R-square & 0.382 & 0.400 & 0.499 & 0.508 \\
\hline
\end{tabular}

Parameter estimates for RUG-III/22 groups and ward identifiers are not shown.

$* p<0.05$.

$* * p<0.01$.

$* * * p<0.001$.

age and LOS were not statistically significant at the 0.05 level.

Table 2 presents four models for calculating cost weights. In the basic non-adjusted model, the cost weights for RUG-III/ 22 spanned a six-fold range from $\mathrm{PA}=0.42$ (low) to $\mathrm{RFC}=2.52$ (high). The range between the least costly and most costly group increased somewhat in the three adjusted models. In the non-adjusted RUG-III/22 model five groups (PA, $\mathrm{BA}, \mathrm{IA}, \mathrm{CA}$ and CB) had a higher coefficient of variation $(\mathrm{CV})$ of resource use than that of the whole sample (0.65). These less homogeneous groups were also the least costly. A majority $(75 \%)$ of the group means differed statistically $(p<0.05)$ from each other; when differences were not significant, one group was usually represented by only a few observations. Also, some overlap of cost weights was expected, as groups represent clinically different residents. The mean nonadjusted cost weight for residential home residents was 0.82 , and for health centre hospital residents 1.15 . Thus, the health centre residents were on average almost 30\% more cost intensive than residential home residents. Within each RUG-III/22 clinical category residents of residential homes, not shown, were more concentrated into the lower ADL dependency groups than those of health centres.

\section{DISCUSSION}

A simple algorithm of the Resource Utilization Groups, Version III appears to be a valid and reliable system in Finnish long-term care facilities. The interrater reliability of RUG-III/22 assessments was high, especially for crucial classification items, such as ADLs, and when comparing cost weights. The low kappa values found in memory/recall ability in the comparison of a personal nurse and outsider evaluator could be due to the fact that the ability to remember can be highly variable for persons with impaired cognitive skills and may therefore be difficult to assess by an outsider evaluator. Our sample size did not allow assessment of inter-rater reliability of all individual RUG-III/22/MDS variables. Extensive reliability estimates of MDS items have been performed in the USA and in other countries $(11,16)$.

The adapted RUG-III/22 classification alone explained a considerable amount of variation in cost $(38.2 \%)$, comparable to that found in other countries. The exclusion of short-term wards may have resulted in a lower variance explanation and a lower proportion of rehabilitation residents. Resource use within groups was relatively homogeneous and the fact that the low cost groups were more heterogeneous also corresponds to results of other derivation and validation studies (4-7). The merging of original RUG-III groups to form the 22-group model did not significantly affect the variance explanation and the homogeneity of groups. The adjustments and exclusion of groups in the "special rehabilitation" category were made based on Finnish practice patterns and the study sample. Whether the current provision of rehabilitation is adequate and effective could not be determined.

The cost weights of the adapted RUG-III/22 followed logical patterns increasing with higher dependency in ADLs. Health centre residents were as expected more costly than residential home residents. The cost weights computed for RUG-III/22 groups were based on relatively large group sizes. In only three groups were the number of observations less than 20 . In particular, the cost weight for SE2, with only four observations, must be regarded highly unstable. Overall, the cost weights show patterns similar to those computed in other countries. Consistency of direct mean care time by RUG-III groups between five countries has been previously shown by Carpenter et al. (17). We do not know the potential bias caused by 
including two large facilities, and only those in urban areas. It did not appear that staff perceived the project as a "time and motion study" and that this influenced management decisions on staffing levels. Feedback sessions indicated that the amount of training given for collecting the data was sufficient in most, although not all, cases.

The fact that age, gender and length of stay (LOS) were found to be statistically significant can be regarded as reasonable. It is plausible to assume that residents entering a facility receive more care time, controlling for case-mix, during the first few weeks due to greater service needed for "settling in". Younger residents ( $<75$ years) may be more costly because of greater rehabilitation potential. It is not clear why females would be more costly than men, despite the fact that the vast majority of nursing home residents are female. If age, gender and LOS were to be used as separate outputs (e.g. for payment), it would be well founded to use the cost weights that adjust for these three variables. If ward differences express productivity differences, it would be reasonable to use cost weights adjusting for different staff patterns. On the other hand, different staff patterns may have also expressed different quality and outcomes of care, about which we had no information. Overall, the adjusted RUGIII/22 cost weights did not differ greatly from the non-adjusted.

In conclusion, the results of this study support previous evidence on the transferability of the RUG system between healthcare systems. There appears to be good evidence of the feasibility of using the RUG classification in Nordic settings, building upon the work with the earlier version of RUGs (RUG-II) in longterm care facilities in Sweden (8). The RUG-III/22 model presented in this paper could be especially useful in applications such as information systems, as it requires fewer classification items.

\section{ACKNOWLEDGEMENTS}

We acknowledge the work of the Stakes' (National Research and Development Centre for Welfare and Health) project group including Timo Karjalainen, Annikki Korhonen, Mikko Nenonen, Anja Noro, Olli Nylander, Marja-Leena Perälä, Pekka Rissanen, Reijo Salmela, Reijo Tilvis, Matti Vuoristo, Mats Brommels and the administration and staff of the long-term facilities participating in this effort. The study was performed in collaboration with members of interRAI. The study was supported in part by the University of Kuopio, Academy of Finland, Yrjö Jahnsson Founda- tion, Päivikki and Sakari Sohlberg Foundation and interRAI.

\section{REFERENCES}

1. Fetter RB, Brand DA, Gammache D. DRGs: Their Design and Development. Ann Arbor, Michigan: Health Administration Press, 1991.

2. Fries BE, Cooney LM. Resource Utilization Groups: a patient classification for long-term care. Med Care 1985; 23: $110-22$.

3. Schneider DP, Fries BE, Foley WJ, Desmond M, Gormley W. Case mix for nursing home payment: Resource Utilization Groups, version II. Health Care Financing Review, Annual Supplement 1988; 9: 39-52.

4. Fries BE, Schneider DP, Foley WJ, Gavazzi M, Burke R, Cornelius E. Refining a case mix measure for nursing homes: Resource Utilization Groups (RUG-III). Med Care 1994; 32: $668-85$.

5. Carpenter IG, Main A, Turner GF. Casemix for the elderly inpatient: Resource Utilization Groups (RUGs) validation project. Age Aging 1995; 24: 5-13.

6. Ikegami N, Fries BE, Takagi Y, Ikeda S, Ibe T. Applying RUG-III in Japanese long-term care facilities. Gerontologist 1994; 34: 628-39.

7. Carrillo E, Garcia-Altes A, Peiro S, Portella E, et al. System for the classification of patients in mid and long-term care facilities: Resource Utilization Groups, version III. Validation in Spain. (in Spanish). Revista de Gerontologia 1996; 6: $276-84$.

8. Ljunggren G, Fries BE, Winblad U. International validation and reliability testing of a patient classification system for long-term care. Eur J Gerontol 1992; 1: 48 - 59.

9. Frijters D, Van der Kooij C. Resource Utilization Groups for Nursing Home Patients in the Netherlands: SIG, Dutch Centre for Health Care Information, Utrecht, 1991.

10. Morris JN, Hawes C, Fries BE, et al. Designing the National Resident Assessment Instrument for nursing homes. Gerontologist 1990; 30: $293-307$.

11. Hawes C, Morris JN, Phillips CD, Mor V, Fries BE, Nonemaker S. Reliability estimates for the Minimum Data Set for nursing home resident assessment and care screening (MDS). Gerontologist 1995; 35: $172-8$.

12. Jónsson PV. Letter from Reykjavik. Annals of Internal Medicine 1998; 128: $941-5$.

13. Aro S, Noro A, Salinto M. Deinstitutionalization of the elderly in Finland 1981-91. Scand J Soc Med 1997; 25: $136-43$.

14. Fleiss JL. Statistical methods for rates and proportions. $2^{\text {nd }}$ edn, New York: John Wiley \& Sons, 1981.

15. Fleiss JL, Cohen J. The equivalence of weighted kappa and the intraclass correlation coefficient as measures of reliability. Educ Psychol Meas 1973; 33: 613-19.

16. Sgádari A, Morris JN, Fries BE, et al. Efforts to establish the reliability of the Resident Assessment Instrument. Age Aging, 1997; Suppl 26: 2:27-31.

17. Carpenter GI, Ikegami N, Ljunggren G, Carrillo E, Fries BE. RUG-III and Resource allocation: Comparing the relationship of direct care time with patient characteristics in five countries. Age Aging 1997; 26: 61-5. 